\title{
Reflexividad y Didáctica: Pensamiento que Cualifica la Acción; Acción que Cualifica al Sujeto
}

\author{
Juan C. Molina ${ }^{1}$ \\ José F. González²
}

\section{Resumen}

Este artículo presenta un enfoque desde el cual se propone que la didáctica de los procesos de enseñanza-aprendizaje privilegie la articulación de los contenidos académicos, es decir, el conocimiento formal, con el mundo de la vida cotidiana, en el cual se inscriben los proyectos de vida de los estudiantes. Según esta propuesta, el "recurso didáctico" más importante en el proceso de enseñanzaaprendizaje lo constituyen las propias vivencias de los sujetos. Sin embargo, su potencial como portadoras de conocimiento sólo puede emerger gracias a la reflexión que el estudiante haga sobre ellas, en función de sus intereses formativos. Esta clase de reflexividad puede convertir la vivencia en experiencia, la cual no sólo es portadora de conocimiento, sino que también da sentido a éste, en la perspectiva del proyecto de vida del estudiante. Todo aprendizaje por vía de la experiencia es, en consecuencia, un aprendizaje significativo para la formación del sujeto. De igual manera, la reflexividad como estrategia didáctica, incluye al docente como sujeto que reflexiona sus prácticas de enseñanza, por lo cual, en rigor, también es un sujeto en formación.

1 Facultad de Ciencias, INSTITUTO TECNOLÓGICO METROPOLITANO, juanmolina@itm.edu.co

2 Facultad de Comunicación Audiovisual, Politécnico Colombiano Jaime Isaza Cadavid, jfgonzalez@elpoli.edu.co

Fecha de recepción: 17 de Agosto de 2010

Fecha de aceptación: 10 de Noviembre de 2010 


\section{Palabras clave}

Aprendizaje significativo, didáctica, experiencia, reflexividad, vivencia.

\section{Abstract}

This paper establishes an approach which proposes that didactics in the education-learning processes favor the formal knowledge articulation which configures the academic world, with the daily life, in which the students' life projects are registered. From this point of view, the most important "didactic resource" in the education-learning process constitutes it the subjects' own livings. However, their potential as knowledge carriers only can emerge by the students' reflection about them. This reflexivity such as didactic strategy can become the living in experience, which is not only a knowledge carrier, but also gives them sense, in the learners' life project perspective. Any learning process by experience means is, in consequence, a significant learning for the student training. At the same time, reflexivity as a didactic strategy involves the teacher as a subject who evaluates his educational practices, that's why he is also a learner.

\section{Keywords}

Didactics, experience, meaningful learning, reflexivity. 


\section{INTRODUCCIÓN}

En la búsqueda de propuestas didácticas que sean coherentes con la visión de la educación concebida como búsqueda y encuentro con la verdad, - lo cual incluye el encuentro consigo mismo-, que promuevan el mejoramiento de las relaciones pedagógicas y que orienten los procesos de enseñanza-aprendizaje hacia la generación de aprendizajes significativos para el desarrollo humano, se plantea el valor de la reflexividad como estrategia didáctica, cuyo auge en la actividad educativa colombiana va en aumento, aunque aún esté lejos de consolidarse como una práctica generalizada en los escenarios de todo el sistema educativo.

La pretensión concreta con una estrategia didáctica reflexiva, radica en poder lograr no sólo algunos objetivos de aprendizaje de los contenidos curriculares, sino que sean significativos y relevantes en la construcción de la subjetividad e identidad del estudiante, tanto en su dimensión cognoscitiva como socioafectiva.

En este texto, se trata de explorar una propuesta didáctica en la cual el docente anima a sus estudiantes a reflexionar sobre algunas de sus vivencias para tomar conciencia crítica de ellas y convertirlas en experiencias portadoras de saber, asumiendo aquí que el saber es conocimiento dotado de sentido. Se concibe aquí la categoría vivencia como un suceso significativo desde el punto de vista afectivo en la vida del sujeto, pero que, no obstante su potencial relevancia, no ha sido integrado como parte de algún conocimiento formal, toda vez que conserva su naturaleza espontánea, intuitiva e irreflexiva, propia de la cotidianidad. Se ubica en un momento histórico del mundo de la vida, que se caracteriza por ser, en palabras de Mèlich (1994), "la esfera, el horizonte espacio-temporal en el que transcurren las vivencias, pensamientos y acciones humanas de orden espontáneo o irreflexivo [...] el mundo intuitivo, pre-racional, [...] en el que nuestros actos tienen lugar maquinalmente, dado que muy pocas veces actuamos racionalmente en la cotidianidad. [El mundo en el que] nos limitamos a vivir, no a pensar que vivimos".

Adicionalmente, en esta conceptualización se consideran también como vivencias, y de forma no menos importante, algunas 
actividades prácticas de carácter "realista", planeadas por el docente con el fin de que el estudiante acometa ciertas tareas de manera más o menos espontánea, es decir, con relativamente pocos elementos teóricos.

Luego, la reflexión intelectualmente rigurosa del sujeto sobre sus vivencias y acciones, daría lugar a un conocimiento teóricopráctico que no es otra cosa que la cualificación de tales vivencias en experiencia, como un saber que retorna articulándose de manera significativa al proyecto de vida del aprendiz, cualificándolo. En este orden de ideas, además, el docente cuando reflexiona sobre sus prácticas de enseñanza —al fin y al cabo vivencias, también- las convierte en experiencias, es decir, en saber teórico-práctico sobre su didáctica, lo que constituye, dicho sea de paso, la cualificación de su práctica docente.

Por otra parte, es necesario precisar el concepto de reflexividad adoptado en esta propuesta, el cual implica, según Dewey (1989), citado por Hoyos (2006) "el examen activo persistente y cuidadoso de toda creencia o supuesta forma de conocimiento a la luz de los fundamentos que la sostienen y las conclusiones a las que se tiende". Por lo tanto, asumida como estrategia didáctica, la reflexividad debe estar planificada y orientada hacia los objetivos de aprendizaje definidos por los currículos.

Adicionalmente, agrega Hoyos que a lo largo de la historia de las prácticas educativas, el concepto reflexión ha pasado por diversas descripciones, y se han identificado múltiples funciones en los procesos de enseñanza-aprendizaje, entre las cuales se destaca "su función como categoría del pensamiento interactivo" (Hoyos, 2006). Esto implica que se debe planificar y ejercer la reflexión de carácter didáctico sobre las vivencias, de dos maneras particulares: una intra-subjetiva, que es la que hace el sujeto cuando dialoga consigo mismo, y otra inter-subjetiva, cuando interactúa con los otros estudiantes con quienes comparte el proceso de enseñanza-aprendizaje. De esta manera las experiencias y pensamientos de docentes y estudiantes inmersos en los procesos de enseñanza-aprendizaje del grupo de aula y las diferentes visiones del mundo que emergen en el proceso, se convierten en referentes e insumos de aprendizajes significativos relevantes para sus proyectos de formación, cuya manifestación 
concreta se evidenciaría en la constante transformación y cualificación de sus actuaciones futuras.

Es importante agregar que la reflexividad inter-subjetiva al vincular el mundo académico con el mundo de la vida de los integrantes del grupo, puede propiciar y consolidar al interior de la comunidad académica del aula un tejido sociocultural de gran riqueza, atravesado y soportado en el respeto por el otro y por la reciprocidad, esta última, categoría pedagógica clave en la interacción comunicativa. Cabe precisar que aquí reciprocidad se concibe como una genuina predisposición al diálogo crítico, proclive al disenso más que al consenso, aunque este último ha de ser muy valorado cuando es auténticamente construido, y no impuesto. Max Weber, citado por Mèlich (1994), advierte que "no se puede confundir la reciprocidad con la homogeneidad de significaciones, ni menos aún con la solidaridad, la cooperación o la fraternidad". De hecho, la reciprocidad es la actitud que hace posible pensar los conflictos sociales como recursos pedagógicos y oportunidades para el aprendizaje. Según Weber "el conflicto es un modo de encuentro social (y pedagógico) de primera magnitud" (Mèlich, 1994).

\section{LA FORMACIÓN COMO RESPONSABILIDAD DEL SUJETO}

Desde la perspectiva educativa, es posible diferenciar a un sujeto que se asume a sí mismo como proyecto en formación de otro que se pone en manos del docente y el currículo. Esta distinción radica en que el primero intenta "aprender a aprender", es decir, a construir el conocimiento por sí mismo, en tanto que el segundo necesita ser instruido y entrenado con lo necesario para ser funcional en contextos predeterminados, especialmente en los del mundo laboral. El primero se piensa a sí mismo problemáticamente, conflictivamente, en los contextos, y en esa medida deberá aprender a resolver problemas. El segundo necesita programarse de manera eficiente para resolver lo conflictivo adaptándose miméticamente a las variaciones y contingencias de los contextos. 
El primer sujeto, pues, es consciente de muchas de las necesidades formativas que demanda su proyecto de vida, y de que satisfacerlas depende en gran medida de su propia participación y compromiso, elementos imprescindibles para la construcción de sus aprendizajes. En esta perspectiva Jesús Beltrán (2003) señala que "la calidad del aprendizaje pasa más por la calidad de las acciones del estudiante, que por la calidad de las actividades del docente", razón por la cual "el aprendizaje es en realidad la huella del pensamiento".

Este estudiante requiere que el diseño didáctico de su proceso educativo privilegie las estrategias de aprendizaje, que son las que de manera más contundente contribuyen con el desarrollo de la capacidad para aprender autónoma y eficazmente. Estas estrategias generalmente son actividades intelectuales de naturaleza metacognitiva y crítica, que implican necesariamente la reflexión y evaluación constantes del estudiante acerca de sus actuaciones orientadas a aprender, y también de otras de las que podría decantar conocimientos relevantes para sus intereses. Cabe destacar que el tipo de pensamiento meta-cognitivo resulta clave en esta propuesta, dado que "involucra un proceso de conocimiento consciente que genera sentido y modifica las estructuras cognitivas previas para generar un nuevo proceso de aprendizaje, de aplicabilidad $y$ de modificabilidad del comportamiento del individuo en una cultura" (Muñoz, 2006).

Adicionalmente, un sujeto que asume los procesos de aprendizaje como medios para satisfacer sus intereses e inquietudes académicas y formativas, y para potencializar las destrezas necesarias para poder concretar su proyecto de vida, es alguien que, como afirma Mèlich (2002), "tiene inherente a su condición la obligación de inventarse, de construirse, de llegar a ser".

Esta actitud participativa e interesada del sujeto en su propia formación puede denominarse voluntad de ser. Ésta a su vez la expresa aquél a través de sus interacciones al interior del contexto sociocultural en el que está inmerso, lo que le implica una actitud de lectura e interpretación constantes de dicho contexto, es decir, una actitud que se constituye en voluntad de saber. En consecuencia, en tanto que está inmerso dentro de esa realidad 
que lee e interpreta, el sujeto es objeto de conocimiento de sí mismo. Por lo tanto esta voluntad de saber, es también voluntad de saber-se.

De esta manera, el estudiante con genuinos deseo y voluntad de ser y saber, concibe su aprendizaje como la vía principal para conquistar aquello que quiere llegar a ser y hacer; y en la medida en que lo vaya logrando, estará construyendo su proyecto de vida, es decir, autorrealizándose.

A través de estas estrategias el estudiante puede autorregular su proceso de aprendizaje, y a la postre, estas capacidades para adquirir conocimientos y para reflexionar sobre sí mismo, son las que le permitirían anticipar, explicar y controlar la naturaleza, y paralelamente construir su dimensión cultural (Rodríguez, 2006).

\section{CONCEPCIONES DIDÁCTICAS}

\subsection{Entre la Instrucción y la Comprensión}

En términos generales, la didáctica como saber práctico trata de responder a la pregunta de "cómo enseñar todo a todos", y se asocia naturalmente con el campo práctico de la enseñanza. Como disciplina, parte de principios teóricos, modelos comprensivos, reglas prácticas, métodos y estrategias articuladas. Resulta lógico, pues, que se le defina como una disciplina aplicada consistente en el desarrollo de métodos y técnicas de enseñanza que, con frecuencia, se configuran como "kits didácticos" cuyos objetivos son la eficacia y la eficiencia en la enseñanza de conocimientos específicos pertenecientes a campos disciplinares y/o a alguna ciencia en particular. De hecho, es muy común la creencia de que cada campo del conocimiento tiene su propia didáctica, noción de la que derivan ideas como la de una "didáctica de las ciencias", o como la de la "tecnología educativa", según la cual, la didáctica se reduce a un saber de tipo procedimental, suficiente para la enseñanza que consiste en el puro entrenamiento. Esta visión acrítica es característica del tipo de educación puramente instruccional. 
En este texto, sin embargo, la intención es vislumbrar otra manera de concebir la didáctica que trascienda la concepción meramente instrumental. Se propone aquí una actuación didáctica concebida a partir de la valoración del docente sobre las relaciones pensamiento-acción del estudiante, toda vez que las actuaciones del ser humano se producen, predominantemente, en función de los significados que atribuye a las situaciones que ha vivido previamente. Dicho de otra manera, de entre todas sus vivencias, aquellas que tengan un significado importante para él, sobre todo desde el punto de vista afectivo, se podrían convertir en experiencias portadoras no sólo de conocimiento sino también de sentido, el cual retroalimentaría y cualificaría el pensamiento con el que comandaría sus próximas actuaciones. Así puede generarse una espiral de aprendizajes significativos, equilibrada por la articulación pensamiento reflexivo-actuación, logrando una relación dinámica y productiva entre teoría y práctica.

En este sentido, "la reflexión como un recurso didáctico permite entender la didáctica, no de modo técnico, sino como proceso articulador de la teoría y de la práctica" (Hoyos, 2006) que trascienda lo puramente instrumental, y que vincule el conocimiento académico con la red de significados de las vivencias tanto del docente como de sus estudiantes (Feldman, 1999). Esta concepción de la didáctica le apuesta a una educación que satisfaga la "necesidad de entendimiento" (Max-Neef, 1986) del estudiante, aprovechando el hecho de que "la reflexión sobre las vivencias constituye una vía de comprensión” (Hoyos, 2006).

\subsection{Reflexividad para el Aprendizaje Significativo y el Desarrollo Humano}

Las experiencias, pues, son eventos de aprendizaje con sentido que transforman y enriquecen la subjetividad e identidad del sujeto; en otras palabras, son aprendizajes significativos. Así mismo, estos son los acontecimientos de que está hecha la historia subjetiva del "llegar a ser"; son hitos del desarrollo humano y de la auto-realización del sujeto.

Parafraseando a David Ausubel, el aprendizaje significativo puede darse siempre y cuando aquello que se necesita y desea aprender, se pueda poner en relación, de forma intencional y no 
por azar ni aleatoriamente, con los saberes previos del estudiante, siempre que éste tenga la disposición de ánimo para aprender de esa manera (Ausubel, 1983). Añade el autor que el aprendizaje es significativo toda vez que se integre a las estructuras cognoscitivas con las que ya contaba el estudiante, particularmente si se produce la integración de sus dimensiones lógica, cognoscitiva y afectiva en la construcción del conocimiento. En la misma dirección, Hernández (1998) afirma que "para que el aprendizaje significativo se logre, es necesario que en primer lugar sea autoiniciado y que el alumno vea el tema, el contenido o los conceptos que se van a aprender como algo importante para sus objetivos personales ([en otras palabras,] como algo significativo para su desarrollo y enriquecimiento personal)".

En efecto, si el punto de partida de los estudiantes para el aprendizaje del conocimiento formal es usar la reflexión sobre las vivencias que han tenido en los contextos en que interactúan cotidianamente, el conocimiento científico que puedan adquirir tendrá sentido para sus proyectos de vida. Dicho de manera esquemática, el estudiante debe reflexionar sobre su trayecto de vida en función de su proyecto de vida, ya que "la reflexión implica la toma de conciencia del modo como opera la cotidianidad y de la influencia que tiene la historia de cada sujeto en su construcción" (Hoyos, 2004). Esta manera de concebir la reflexividad como estrategia didáctica, se apoya en el hecho de que "es en la cotidianidad [...] que el sujeto tiene conciencia de la existencia del mundo y de los diversos escenarios en los que el mundo existe para él, [...] [y porque es allí] donde se producen las experiencias”.

Se parte de que en general el aprendizaje puede producirse en una dinámica de actuación-reflexión, de cuya rigurosidad depende el nivel de complejidad y relevancia del conocimiento alcanzado, en un círculo hermenéutico de significación y re-significación que ha de traducirse finalmente en aprendizajes significativos de los estudiantes y, eventualmente, en su desarrollo humano. Finalmente, un aprendizaje significativo generador de desarrollo, es aquel que "propicia el despliegue de configuraciones de excelencia en el sujeto", y es el tipo de aprendizaje "mediante el cual el sujeto se apropia de contenidos (conocimientos, autoconocimientos, sentimientos, actitudes, valores, formas de 
relacionarse) actuales y potenciales", desde los cuales puede adoptar sólidos puntos de vista "que le posibilitan actuar acertadamente, transformar y crear en diferentes contextos" (Cartaya, 1998), conforme va construyendo su proyecto de vida.

\section{LA DIDÁCTICA REFLEXIVA: PROPUESTA PRÁCtICA}

La primera inquietud que aparece cuando el docente se decide a adoptar la estrategia de la didáctica reflexiva, está referida a saber cuándo es oportuno animar y promover los procesos de reflexión para el aprendizaje. Antes de abordar esta pregunta y otras de igual importancia, es necesario establecer como referente general que, como afirma Feldman (1999) de las vivencias se puede extraer conocimiento desde tres perspectivas: " $l$ conocimiento en la actuación, la reflexión en la actuación y la reflexión sobre la actuación".

Sin embargo, tanto el conocimiento que está contenido en la actuación, como el producto concreto de la reflexión, tanto en el preciso momento como después de la vivencia o actuación, no "existen" mientras éstos no se hagan explícitos, bien oralmente o por escrito. Esto implica para el docente una planeación estratégica de algunas actividades orientadas a que los estudiantes hagan explícitas sus vivencias o actuaciones para poder reflexionar didácticamente sobre ellas. Para comprender mejor la manera en que puede hacerlo, es conveniente analizar los dos tipos de vivencias o actuaciones que en general, según la estrategia didáctica que se ha propuesto, serían objeto de reflexión.

En primer lugar, están las vivencias cotidianas pertenecientes a la historia personal de los estudiantes. En cuanto a éstas, el primer momento de reflexión se produciría cuando el docente anime a los estudiantes a buscar y seleccionar una vivencia personal afectivamente significativa en su historia. La tarea consiste en narrarla y/o describirla de manera autobiográfica, induciéndose necesariamente durante su producción la reflexión interior (intra-subjetiva) del estudiante. Con este ejercicio se trata de "retrotraer" algunas vivencias de los sujetos que pudieran 
servir, entre otras cosas, para ilustrar, comparar y/o contrastar el "contenido" de la experiencia con los contenidos teórico-prácticos del curso. Adicionalmente, si estas experiencias son compartidas en el grupo a través de su lectura en el aula, se constituyen en los pre-textos necesarios para propiciar, a través de actividades participativas de discusión, procesos de reflexión colectiva (intersubjetiva).

Otra actividad similar que se sugiere, es la de realizar talleres de reflexión sobre vivencias más o menos comunes a todos, (como por ejemplo acerca de las formas de comunicación y los métodos de enseñanza de valores en el hogar, las formas de participación en los eventos familiares importantes, algunos sucesos relevantes de la niñez, conflictos importantes con amigos o familiares cercanos, entre otras), diseñados y orientados por el docente, y que puedan vincularse de alguna manera con los contenidos académicos.

Sin embargo, hay que advertir que precisamente por el hecho de que el "aprendizaje llega a ser significativo cuando involucra a la persona como totalidad (procesos afectivo y cognitivo) y se desarrolla en forma experiencial ([es decir,] que se entreteja con la personalidad del alumno)" (Hernández, 1998), con frecuencia durante estos ejercicios reflexivos emergen las emociones de los participantes, a veces con bastante intensidad. Esta situación podría llegar a ser contraproducente, si no se ha construido un clima propicio en el aula. Para aprovechar el potencial didáctico del ejercicio auto-reflexivo así como el interpersonal se requiere entonces la disposición de los participantes para apostarle a esta metodología. Así mismo, un grupo dispuesto, depende en gran medida de que el docente haya desplegado su tacto pedagógico para generar las condiciones apropiadas.

En segundo lugar, están las vivencias o actuaciones de naturaleza propiamente curricular, es decir, actividades de aprendizaje activo orientadas a que el estudiante identifique, coteje y ponga en práctica, en el ámbito del mundo académico y/o profesional, el conocimiento teórico. Entre otros ejemplos, se pueden hacer simulacros, microproyectos de investigación, diseño de propuestas o productos, visitas, pasantías, o entrevistas a expertos. 
Estas actividades deben estar apoyadas en herramientas didácticas como el diario de campo, o registros visuales y/o de audio, entre otras, que permitan la explicitación de la experiencia que luego será objeto de socialización y retroalimentación colectiva, en clave de discusión crítica, con la moderación y orientación del docente.

Por último, es necesario señalar que no existe una fórmula o esquema para el diseño de una estrategia didáctica reflexiva. Esto resultaría absurdo, ya que por definición la didáctica reflexiva es una manifestación de pensamiento crítico acerca de las fórmulas didácticas pre-concebidas, razón por la cual la planeación didáctica de las actividades reflexivas del curso y su aplicación, dependen mucho de la creatividad del docente y sus estudiantes, así como de diversas circunstancias y características del grupo.

En esta propuesta, no obstante, privilegiar la reflexión por encima de la actuación, pero sin prescindir de ésta, debe ser el criterio rector así como el eje transversal de todo el accionar didáctico.

\section{CONCLUSIONES}

La reflexividad estimula la racionalidad del sujeto, es decir, su capacidad de crear, discurrir y juzgar con fundamento. Esta racionalidad constituye el atributo necesario para construir el conocimiento y comprensión del mundo, la cultura, los fenómenos naturales $\mathrm{y}$ sociales $\mathrm{y}$ sus diversas interrelaciones, $\mathrm{y}$ esta construcción determina la manera de ser, estar y sentir del sujeto en dichos escenarios. Por tanto, el pensamiento reflexivo contribuye al desarrollo de la creatividad y de las capacidades crítica y auto-crítica, bajo el estímulo de procesos metacognitivos y autoevaluativos.

Por otra parte, una comunidad académica de aula en la cual conviven sujetos que deciden poner el acento de su formación en las estrategias de aprendizaje, —es decir, estudiantes en lugar de alumnos - , pueden llegar a configurar, por la vía de la didáctica reflexiva, un tejido de interpretaciones inter e intra-subjetivas a partir de sus experiencias, que haga emerger a cada uno como un 
"otro" distinto y significativo, así como un "yo" re-conocido y en lo posible re-inventado. Se daría, pues, en los estudiantes, a partir del ejercicio intencionado del pensamiento reflexivo como estrategia didáctica, más allá del aprendizaje puramente académico, el reconocimiento del otro y de sí mismo, así como el autodescubrimiento y apropiación conciente de sus saberes previos $\mathrm{y}$, posiblemente, de nuevas potencialidades.

Este es el juego de espejos de la reflexividad: no se es nadie si no hay otro que actúe como imagen refleja, que permita ver e interiorizar lo humano del propio yo. En otras palabras, la reflexividad se configura como un camino para descubrir-se y autoinventar-se, es decir, para "llegar a ser", desde los otros como necesarios puntos de referencia, proceso que requiere al lenguaje y la comunicación como vehículos indispensables.

\section{REFERENCIAS}

Ausubel, D., Novak, J., Hanesian, H., (1983); Psicología Educativa: Un punto de vista cognoscitivo. México: Ed. Trillas. p. 623.

Beltrán, J., Bueno, J.A., (1995); Psicología de la educación. Editorial Boixareu Universitaria: Barcelona. p. 664.

Cartaya, P., (1998); El pensamiento pedagógico de José de la Luz y Caballero. Revista Varona: La Habana.

Dewey, J., (1989); Cómo pensamos. Nueva exposición de la relación entre pensamiento reflexivo y proceso educativo. Barcelona: Paidós. p. 249.

Feldman, D., (1999); Ayudar a enseñar. Relaciones entre didáctica y enseñanza. Buenos Aires: Ed. Aique. p. 95-102.

Díaz, F., Hernández, G., (1999); Estrategias docentes para un aprendizaje significativo: Una interpretación constructivista. Ed. McGraw-HILL: México.

Hernández, G., (1998); Paradigmas en psicología de la educación. México: Paidós. p. 99-115.

Hoyos, A.J., (2006); Formación y proyecto: una propuesta didáctica reflexiva para maestros. Tesis doctoral en Ciencias Pedagógicas. Cuba: Universidad de La Habana. 
Hoyos, A.J., (2004); Teoría-práctica, pensamiento-acción: necesarias articulaciones de la acción didáctica. En: Revista Universidad de Medellín; Vol. 39 No 78. Julio-Diciembre de 2004. p. 72-78.

Max-Neef, M. et. al., (1986); Desarrollo y Necesidades. Segunda parte. En: Desarrollo a escala humana. Development Dialogue, Número especial. Suecia: Fundación Dag Hammarkskjold.

Mèlich, J.C., (1994); Del extraño al cómplice: La educación en la vida cotidiana. Barcelona: editorial Anthropos. p. 202.

Mèlich, J.C., (2002); Filosofía de la finitud. Barcelona: Empresa Editorial Herder S.A. p. 44-45, 184.

Muñoz, M.T., (2006); Implicancias de la metacognición en el proceso educativo. Recuperado julio 30 de 2010 de http://www.psicologiacientifica.com/bv/psicologiapdf-123implicancias-de-la-metacognicion-en-el-procesoeducativo.pdf

Rodríguez, B.A., (2006); La didáctica basada en procesos, una alternativa para innovar las prácticas educativas en la educación superior. Recuperado el 15 de julio de 2009 de http://www.sadpro.ucv.ve/Ponencias/blanca-de-escontrela.pdf

Shütz, A., Luckmann, T., (1973); Las estructuras del mundo de la vida. Buenos Aires: Amorrortu. Pp. 320.

Taylor, C., (1997); Argumentos filosóficos. Barcelona: Paidós. p. 384. 